tion. It is considered unclean by some Hindu women to prepare their husband's food during menstruation; a Moslem woman cannot pray in the mosque when menstruating - a particularly burdensome restraint during the annual feast of Ramadan. A preliminary trial of a "Ramadan pill" in Calcutta in 1976 showed that the concept was understood by poor urban women. ${ }^{8}$ A few Moslem women already use hormonal treatment to postpone the menses when on pilgrimage to Mecca. Some Buddhists think it is wrong to enter a temple during menstruation. Possibly some of the emerging middle classes of the Third World countries might like to change their menstrual patterns for reasons of social convenience as in the West; the cost of sanitary protection is relatively a much greater burden for a woman with limited income. Perhaps other social groups would also like to avail themselves of this opportunity on specific occasions.

Having established that infrequent periods are welcome and attainable, we must now define the optimal dosage of steroids necessary to maintain symptom-free cycles, and establish the most acceptable cycle length. The present tri-cycle regimen is certainly not the final solution to this problem. In view of the acceptable cycle control achieved with a combined pill containing $50 \mu \mathrm{g}$ ethinyloestradiol we have already started a new trial using a pill with only $30 \mu \mathrm{g}$ of this oestrogen. Possibly the oestrogen dosage may even be further reduced in the latter weeks of each three-monthly cycle without sacrificing good cycle control.

These developments are part of the inevitable pattern of evolution of hormonal contraception, but any change in dosage regimen will require its own careful epidemiological assessment. If reduction of menstruation proves to be generally acceptable, it opens up a whole range of new and hitherto unexplored approaches to contraceptive development.

We thank R D Slacke of Organon Laboratories Ltd for preparing the special packs of pills and printing the instructions for this trial, and the staff of the Family Planning Centre for their help.

\section{References}

1 Piotrow, P T, and Lee, C M, Oral Contraceptives Population Report, Series A, Number 1. Washington, Publications of the Population Information Program, Dept of Medical and Public Affairs, The George Washington University Medical Centre, April 1974.

2 Short, R V, Proceedings of the Royal Society. Series B. Biological Sciences, 1976, 195, 3.

${ }^{3}$ Short, R V, in Physiology and Genetics of Reproduction, part A, ed E M Coutinho and F Fuchs. New York, Plenum Publishing Corporation, 1974.

4 World Health Organisation, Expanded Programme of Research, Development and Research Training in Human Reproduction. Fourth Annual Report. Geneva, WHO, 1975.

5 Lydecken, K, Acta Prychiatrica Scandinavica, 1972, suppl No 229.

${ }^{6}$ Miller, W B, and Smith, P J, Fournal of Psychiatric Research, 1975, 12 (3), 153.

${ }^{7}$ Koetsawang, S, et al, fournal of the Medical Association of Thailand, 1974, 57 (8), 396

${ }^{8}$ Mullick, B, 1977. Personal communication.

(Accepted 23 fune 1977)

\title{
Primary immunisation and febrile convulsions in Oxford 1972-5
}

\author{
PAUL HARKER
}

British Medical fournal, 1977, 2, 490-493

\section{Summary}

A three-year study of febrile convulsions in Oxford with comprehensive notification from general practice and hospitals showed a $3 \%$ risk for all children of suffering at least one febrile convulsion by the age of 5 years. Children were most at risk between 6 and 27 months, and febrile convulsions were most likely to be prolonged in children aged 9-15 months.

The association between febrile convulsions and primary immunisations in the preceding 28 days was compared in case and control children, matched for age and sex. Results suggested that such association was a chance relationship with age. If association was direct, the febrile convulsion rates per 1000 immunisation doses were estimated as follows: diphtheria, pertussis, tetanus -0.09 per 1000; poliomyelitis -0.6 per 1000 ; and measles -0.9 per 1000 . Hence if any of these vaccines had a specific causal relationship with febrile convulsions, these rates would probably have been much higher.

Oxford Area Health Authority (Teaching), Oxford OX1 1LE PAUL HARKER, MB, MFCM, community physician (child health)

\section{Introduction}

Prospective studies of birth cohorts have shown that $2-4 \%$ of children experience at least one febrile convulsion before 7 years of age. ${ }^{12}$ Epilepsy may develop in $2 \%$ of children who have had febrile convulsions. ${ }^{2}$ A causal link between prolonged febrile convulsions and epilepsy has been postulated. ${ }^{3}$ Febrile convulsions occur most commonly between the ages of 6 months and 3 years. Primary immunisation of children takes place during this period and at least two types of immunisationspertussis and measles-may potentially be accompanied by febrile and neurotoxic side effects. Surprisingly little is known about the association of primary immunisation with febrile convulsions.

In Millichap's book on febrile convulsions ${ }^{4}$ a review of studies suggests an association with immunisation in $0.7 \%$ of episodes, with a range from $1-4 \%$ in seven of 33 studies in which an association with immunisation was reported. These studies were of different type in different parts of the world between 1929 and 1964, and so a combined rate is relatively meaningless. None of the studies were from the United Kingdom. Landrigan and Witte ${ }^{j}$ summarised nine years' experience of measles immunisation in the United States. Eleven children in a population for whom 50.9 million doses of vaccine were distributed suffered febrile convulsions 5-15 days after immunisation-an incidence of $0 \cdot 2$ per million doses.

Pertussis immunisation has not been linked specifically with febrile convulsions. Kulenkempff et $a l^{6}$ reported neurological complications after pertussis immunisation in 36 
children aged 3-18 months. Thirty-two developed convulsions that were not categorised, and four developed infantile spasms. In one-third of these cases there were concurrent factorsprevious history of fits; family history of fits in first degree relatives; previous neurodevelopmental defect; previous reaction to immunisation; or recent intermittent infection. These were suggested contraindications to pertussis immunisation.

Melchior ${ }^{7}$ reported no change in the age of onset of infantile spasms in Denmark at periods when whooping cough vaccine was given at 5,6 , and 15 months of age and at 5 and 9 weeks and 10 months of age. He concluded that although there may be occasional connection between immunisation and infantile spasms, the most important factor was a coincidence between immunisation given at a certain age and the neurological disorders that are natural for that age.

Stewart ${ }^{8}$ described a pertussis reaction syndrome from a study of 160 cases of complications associated with pertussis immunisation. This syndrome included the following features: "marble pallor, rigidity, unresponsiveness, altered response to the parents, one or more convulsions with or without pyrexia and cyanotic episodes (blue fits), infantile spasms extending into convulsions." Stewart suggested that the prevalence of the pertussis reaction syndrome might be as high as 1 in 10000 .

Previous studies of febrile convulsions have concentrated on hospital admissions only, often many months after the event. In this study notification of febrile convulsions from general practitioners, health visitors, and hospitals was combined with interview of the parents of case and control children matched for age and sex. At the time of the study health visitors conducted routine follow-up of reactions to immunisation occurring within one month. There was therefore a unique opportunity to examine the association of immunisations and febrile convulsions in Oxford children.

\section{Methods}

The three-year study of febrile convulsions was conducted in Oxford from November 1972 to November 1975. Febrile convulsions were defined as observed or reported muscular contractions and loss of consciousness associated with fever in children aged 0-9 years. A convulsion was classified as prolonged if it was a single fit, lasting for 30 minutes or more; or if there were multiple seizures with an interval of 30 minutes or more between the onset of the first fit and the end of the final fit.

General practitioners and health visitors were asked to notify all cases known to them as soon as possible. Additional information was obtained from hospital discharge notifications, immunisation records, and the immunisation reaction surveillance register. After notification a control child matched to each Oxford-born child for age and sex at the time of the first convulsion was selected from the birth register. I then interviewed the parents of patients and controls, usually within six weeks.

When the study began the child population aged 0-5 living within the boundary of Oxford City had been defined as 6617 at the 1971 census. Primary child care was provided by 71 general practitioners, of whom all but three were in group practices. All practices had health visitors attached. In 1972 the immunisation rates (percentage of eligible children aged 1 year who had completed an immunisation course) were $97 \%$ for diphtheria, pertussis, and tetanus; $95 \%$ for poliomyelitis; and $83 \%$ for measles. In Oxford during the three-year study period, the immunisation schedule for children during the first 27 months was diphtheria, pertussis, and tetanus at 4,5 , and 6 months; poliomyelitis at 7,8 , and 9 months; and measles immunisation at 10 months. The schedule for the last nine months was diphtheria, pertussis, tetanus, and poliomyelitis at 4,6 , and 12 months; and measles at 13 months.

\section{Results}

One hundred and sixty-seven febrile convulsion episodes were reported in 122 children aged 0-10; 160 episodes occurred in 117 children aged 0-5 (see fig). The risk for children of suffering at least one febrile convulsion by the age of 5 years was calculated by taking the

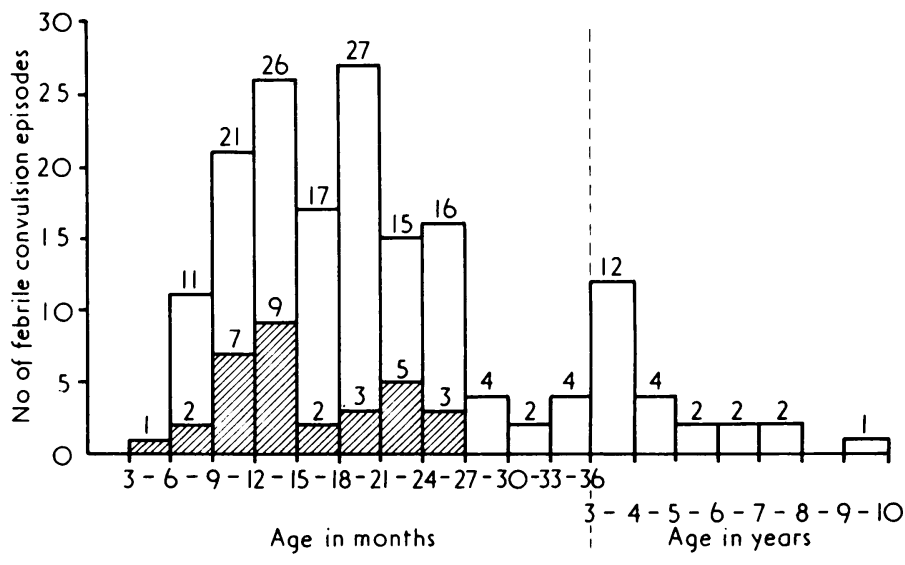

Number of brief and prolonged (hatched areas) febrile convulsion episodes in Oxford children from November 1972 to November 1975 according to age.

number of first fits within $0-5$ population of the 1971 census, and multiplying by $5 / 3$ to equate with a five-year experience. Thus $(117 / 6617) \times(5 / 3)=29$ per 1000 or $2.9 \%$. This is not so accurate as the cohort method, but gives a reasonable approximation assuming that the incidence of febrile convulsions among children resident but not born in Oxford was similar to that among Oxford-born children who had moved from Oxford. The 1971 census probably provided an overestimate of the 0-5 population through to 1975 because there was a fall in birthrate.

There were 32 prolonged episodes $(20 \%)$ in 29 children $(25 \%)$ Forty-two episodes (four prolonged and 38 brief) were not admitted to hospital. When prolonged and brief fits were compared by occurrence at ages below and above 15 months there was a significant excess of prolonged seizures below 15 months (table I).

The association of immunisation with febrile convulsions is shown in table II. An interval between immunisation and febrile convulsion of up to 28 days was included for patients; the same interval was included for controls, using the date of their matched patient's convulsion. This tests the possibility that date of fit may be associated with preceding immunisation by a chance association of time. Only 101 patients were matched with paired controls. One association of measles occurred in a 2-year-old child who had a febrile convulsion six days after measles immunisation. There was no paired control for this child. Numbers of discordant associations with immunisation were too low to merit testing for significance.

TABLE I-Incidence of prolonged and brief febrile convulsions by age below and above 15 month

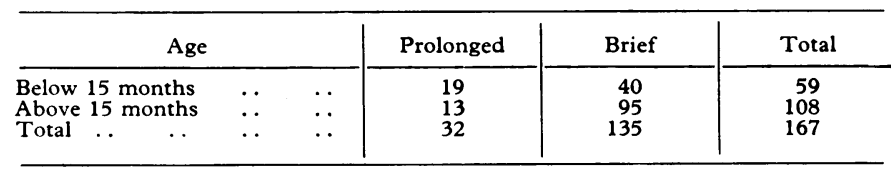

$\chi_{1}^{2}=8.75 ; \mathrm{P}<0.01$.

TABLE II-Association between immunisation and febrile convulsions within 28 days. Absence of data indicates no association

\begin{tabular}{|c|c|c|c|c|c|c|c|}
\hline \multirow{2}{*}{\multicolumn{3}{|c|}{ Immunisation }} & \multirow{2}{*}{$\begin{array}{l}\text { Case } \\
\text { No }\end{array}$} & \multicolumn{2}{|c|}{ Patients } & \multicolumn{2}{|c|}{ Controls } \\
\hline & & & & $\begin{array}{c}\text { Age } \\
\text { (months) }\end{array}$ & $\begin{array}{c}\text { Interval } \\
\text { (days) }\end{array}$ & $\begin{array}{c}\text { Age } \\
\text { (months) }\end{array}$ & $\begin{array}{l}\text { Interval } \\
\text { (days) }\end{array}$ \\
\hline $\begin{array}{l}\text { Diphtheria, pe } \\
\text { tetanus }\end{array}$ & $\begin{array}{l}\text { rtussis, } \\
\ldots\end{array}$ & & $\begin{array}{l}1 \\
2\end{array}$ & 6 & 23 & 7 & 25 \\
\hline Poliomyelitis & . & .. & $\begin{array}{l}1 \\
2 \\
3 \\
4 \\
5 \\
6 \\
7 \\
8 \\
9\end{array}$ & $\begin{array}{r}8 \\
10 \\
11 \\
8 \\
9 \\
11 \\
18\end{array}$ & $\begin{array}{r}12 \\
11 \\
23 \\
7 \\
9 \\
26 \\
27\end{array}$ & $\begin{array}{r}8 \\
10 \\
11\end{array}$ & $\begin{array}{l}10 \\
24 \\
20\end{array}$ \\
\hline Measles & .. & . & $\begin{array}{l}1 \\
2 \\
3 \\
4\end{array}$ & $\begin{array}{l}12 \\
12\end{array}$ & $\begin{array}{r}6 \\
16\end{array}$ & $\begin{array}{l}12 \\
23\end{array}$ & $\begin{array}{l}11 \\
24\end{array}$ \\
\hline
\end{tabular}


Lumbar punctures were performed on seven patients-the case associated with triple antigen, five of the cases associated with poliomyelitis immunisation, and one of the cases associated with measles immunisation. No abnormalities were detected.

A history of febrile convulsions in parents or siblings was significantly associated with the incidence of febrile convulsions in patients compared with controls (table III). Although it was possible to verify the history of siblings in medical records, it was rarely possible to confirm parental history.

TABLE III-Association of febrile convulsions in patients and controls with positive or negative family history of febrile convulsions

\begin{tabular}{|c|c|c|c|c|c|}
\hline \multirow{2}{*}{\multicolumn{4}{|c|}{ Controls }} & \multicolumn{2}{|c|}{ Patients } \\
\hline & & & & \multirow{2}{*}{ Positive } & \multirow{2}{*}{$\begin{array}{c}\text { Negative } \\
2 \\
82\end{array}$} \\
\hline $\begin{array}{l}\text { Positive } \\
\text { Negative : }\end{array}$ & $\therefore$ & 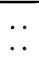 & $\therefore$ & & \\
\hline
\end{tabular}

McNemar's test: $\mathrm{u}=3.1 ; \mathrm{P}<0.01$.

\section{Discussion}

At the end of the study an article in the local paper reported six cases that had not been notified. Some febrile convulsions may not have been reported or may not have been recognised by parents. A rate of $3^{\circ}{ }_{0}$ accords with recent studies. ${ }^{12}$

Febrile convulsions were most common in infants aged between 6 and 27 months, and were most likely to be prolonged between 9 and 15 months. All 10 cases of febrile convulsions associated with immunisation occurred in children aged less than 27 months; eight of these children were aged 9 to 15 months. Was the association a chance finding explained by age ?

\section{DIPHTHERIA, PERTUSSIS, TETANUS}

The total doses of triple antigen were estimated from the number of completed courses reported in 1973. Unfortunately figures for 1974 and 1975 were not available for Oxford alone because of reorganisation of the NHS, but numbers were estimated from the immunisation uptake rates for new Oxfordshire, which were lower than the 1972 Oxford rate. Doses of incomplete courses were not included. The estimate is thus likely to be low, and rates for febrile convulsion associations per 1000 doses of vaccine to be overstated. A linking period of 28 days may also overstate an association.

The rate for diphtheria, pertussis, tetanus was 1 per 10407 doses, that is -0.09 per 1000 doses. Miller et $a^{9}$ found an association of convulsions with pertussis illness of 2 per 1000 children under 5 years of age.

Although doses may be given slightly later than expected from the schedule, an unusually high incidence of febrile convulsions in children aged less than 9 months might be expected in Oxford, as the schedule starts at 4 months, and there is a high rate of uptake. This was not the case. Health visitors recorded a reported history of fever rate after triple antigen of $1 \%$. As many as 104 children might have had fever, and with a febrile convulsion susceptibility of $3 \%$, three children might have been expected to have one or more febrile convulsions. The association in controls is so similar that a chance association with age seems to be the explanation. The one child who had had a febrile convulsion preceded by triple antigen 23 days before had a urinary infection diagnosed after the convulsion.

\section{POLIOMYELITIS}

The rate of febrile convulsions associated with doses of of poliomyelitis vaccine was 7 per 10395 doses, or 0.6 per 1000 doses. The incidence of fits in the 9-12 months age group was higher in this series than expected from other studies. ${ }^{10}$ The interval between immunisation and febrile convulsion, however, was unexpectedly long in three instances. A diagnosis of respiratory tract infection was linked with six of the seven cases, and one case was undiagnosed. Three of the infants $\left(43^{\circ}{ }_{0}\right)$ had prolonged seizures. This is higher than the observed risk of $25^{\circ} \mathrm{o}$ in infants aged $6-12$ months, but the numbers are too small to test significance. Family and birth history of the seven infants were not unusual. Comparison with controls again suggested a chance association with age.

\section{MEASLES}

The rate of febrile convulsions associated with doses of measles vaccine was 3 per 3208 doses, or 0.93 per 1000 doses. Health visitors recorded a history of fever in $13^{\circ}{ }_{0}$ of Oxford children given measles vaccine. Thus 417 children might have had fever. Twelve of these children might have been expected to have associated febrile convulsions. In Miller's study' convulsions were associated with natural measles at a rate of 7 per 1000 illnesses. Two episodes of febrile convulsions in this series were associated with natural measles, both during 1975 when 18 cases of measles in Oxford children under 5 years old were notified. In the three cases immunised within 28 days before a fit there was no personal or family history of fits. A diagnosis of otitis media was made for one child. Comparison with controls excluding one non-paired case showed no significant association.

Measles immunisation is recommended for Oxford children with either a history of convulsions or a family history of convulsions, provided measles immunoglobulin is given at the same time. This study reinforces the view that a child is much more likely to have a febrile convulsion after natural measles than after measles immunisation. Pertussis immunisation is not given to Oxford children if there is a history of fits in the child or family.

\section{FAMILY HISTORY}

Family history is a significant association in many studies of febrile convulsions. ${ }^{10}$ In this study there was no increased association in children who had had prolonged fits. Van den Berg and Yerushalmy ${ }^{11}$ compared retrospective and prospective reports of fits in siblings in families of patients and controls, and found no significant difference. Recall of parental history, however, may be biased towards higher recall in patients' families. The findings of this survey suggest that family history need not be a contraindication, since pertussis immunisation would appear to have neither a direct nor indirect (that is, by inducing fever) causative effect.

Febrile convulsions are the most common type of convulsion in children during the primary immunisation period. In this study 122 children aged $0-5$ years had febrile convulsions in a three-year period. The 0-5 population for England and Wales in 1974 was approximately 3600000 . At a rate of six children affected per $10000-5$ population per year, 21600 children might be expected to suffer at least one febrile convulsion in one year. At a rate of eight episodes per 1000 child population aged 0-5 per year a total of 28800 episodes would occur, half of these (14 400) in the period of immunisation 4-18 months, and one quarter (3600) in this age period would be prolonged. It is therefore an important finding that there was a pronounced lack of association with the components of the primary immunisation course, particularly pertussis and measles, and the association which was found was satisfactorily explained by a coincidence of age. Moreover, no afebrile convulsion during the study period was associated with immunisation.

I thank Dr Christopher Ounsted for his advice and encouragement. 
The study was supported by a grant from the British Epilepsy Association.

\section{References}

1 Van den Berg, B J, Yerushalmy, J, Pediatric Research, 1976, 3, 298.

2 Nelson, K B, Ellenberg, J H, New England fournal of Medicine, 1976, 295, 1029.

${ }^{3}$ Ounsted, C, Lindsay, J, Norman, R, Clinics in Developmental Medicine, No 22. London, Heinemann, 1966.

+ Millichap, J G, Febrile Convulsions. New York, MacMillan, 1968.
5 Landrigan, P J, and Witte, J J, Fournal of the American Medical Association, 1973, 223, 13, 1459.

${ }^{6}$ Kulenkempff, M, Schwartznan, J S, and Wilson, J, Archives of Disease in Childhood, 1974, 49, 46.

' Melchior, J C, Archives of Disease in Childhood, 1977, 52, 134.

${ }^{8}$ Stewart, G T, Lancet, 1977, 1, 234.

${ }^{9}$ Miller, F J W, et al, Growing up in Newcastle upon Tyne. London, Oxford University Press, 1960.

${ }^{10}$ Lennox-Buchthal, M A, Febrile Convulsions: A Reappraisal. Amsterdam, Elsevier Scientific Publishing Co, 1973.

1 Van den Berg, B J, Developmental Medicine and Child Neurology, 1974, 16, 4, 457.

(Accepted 30 fune 1977)

\title{
Family similarities in the age at coronary death in familial hypercholesterolaemia
}

\author{
ARVID HEIBERG, JOAN SLACK
}

British Medical fournal, 1977, 2, 493-495

\section{Summary}

In a combined Norwegian and British study of the age at death from coronary heart disease of heterozygotes for familial hypercholesterolaemia (FH) the correlation coefficients within families for 43 sib pairs was 0.70 and for 14 first cousin pairs 0.61. There was no significant correlation between the age at death and serum cholesterol concentration in either series.

The intrafamilial correlations suggest that information about the age at death from coronary heart disease in heterozygotes within families may have some prognostic value and may also be interpreted as evidence for genetic heterogeneity in FH.

\section{Introduction}

The risk of early death from coronary heart disease in heterozygotes for familial hypercholesterolaemia $(\mathrm{FH})$ is well documented. ${ }^{1-4}$ There is, however, considerable variation in the age at which the first symptoms of ischaemic heart disease are noted or at which coronary death occurs. The reason for such variation may be associated with differences in serum cholesterol concentration ${ }^{5}$ or other risk factors such as blood pressure and smoking, or it may be associated with heterogeneity of the genetic mutations producing the clinical manifestation of $\mathrm{FH}$.

Many clinicians have observed a specially favourable or poor prognosis for $\mathrm{FH}$ within families but few have had the opportunity to observe enough patients to verify this clinical impression. By combining two studies of families with $\mathrm{FH}$, one from Norway and one from England, where the mortality rates from coronary heart disease in the general population are similar, we were able to investigate similarities within families in

\footnotetext{
Department of Medical Genetics, Institute of Medical Genetics, University of Oslo, Oslo
}

\section{ARVID HEIBERG, DRMED, consultant}

MRC Clinical Genetics Unit, Institute of Child Health, London WC1 JOAN SLACK, DM, MRCP, consultant and honorary senior lecturer the age at death due to coronary heart disease among heterozygotes for $\mathrm{FH}$.

\section{Patients and methods}

The data from two family studies of patients with $\mathrm{FH}-$ one Norwegian with 139 kindreds $^{6}$; and one British ${ }^{8}$ with 33 kindredswere combined. All index patients in the kindreds included in this analysis had tendinous xanthomata and hypercholesterolaemia. The cause of death in all patients contributing to these series was verified as being coronary heart disease from death certificates or hospital records.

Altogether 14 of the Norwegian kindreds and eight of the British kindreds contributed to these analyses of the sib-sib correlation coefficients. In addition 14 first cousin pairs were included from the Norwegian kindreds. The cumulative morbidity and mortality rates for the Norwegian and British series of heterozygotes for FH were remarkably similar."

Serum total cholesterol concentrations were determined by standard methods ${ }^{4}$ and adjusted for age and sex as described.

Statistical analysis-Intrafamilial correlations for age at death due to coronary heart disease were calculated for heterozygous sib pairs and cousins. Only relatives of the same generation were used in these calculations to reduce environmental differences which may have contributed to coronary risks. The significance of $r$ values were tested after $\mathrm{z}$ transformation.9 ${ }^{9}$ The combined probabilities for correlation coefficients in sibs and cousins were obtained as recommended by Sverdrup ${ }^{10}$ with tables for small samples. ${ }^{11}$

\section{Results}

\section{AGE AT DEATH}

The mean ages at death in the sibs of probands with $\mathrm{FH}$ who were used for the intrafamilial correlations are shown in table I. The difference in age at death between the Norwegian and British series was significant for men $(P<0.001)$ but not for women. The age at death for women was converted to the equivalent value for men by subtracting nine years, which was the median difference in age at death in the larger Norwegian and British series of which these families were a subsample. The age at death of sibs of the British index patients with $\mathrm{FH}$ is shown in fig 1. More than one mode of distribution was clearly suggested in the British but not in the Norwegian series. The age at death from coronary heart disease in sib pairs is shown in fig 2. A trend for lower age at death in the British than in the Norwegian sibs was apparent.

An intrafamilial correlation for age at death in sibs was also apparent (fig 2) for each of the series as well as for the combined series. The 